\title{
NEW MULTI-BOND ALGORITHM FOR MONTE CARLO SIMULATION OF LATTICE CHAINS
}

\author{
P. RomiszowsKi* aND A. SIKorski \\ Department of Chemistry, University of Warsaw \\ Pasteura 1, 02-093 Warsaw, Poland
}

(Received February 2, 1999; revised version August 3, 1999)

The new algorithm for the Monte Carlo simulations of polymer lattice chains was developed. The model chains were constructed on a simple cubic lattice. The simulations were carried out on chains with and without excluded volume effect using the Metropolis scheme. The basic concept of the new algorithm is the multi-bond modification of the chain conformation instead of applying the classical set of elementary micromodifications. The correctness of the algorithm was verified by studying both static and dynamic properties of the chains. The new algorithm was found to be 3 to 8 times faster than the classical one.

PACS numbers: $02.50 . \mathrm{Ng}, 61.25 . \mathrm{Hq}$

\section{Introduction}

The idea of an algorithm used for the simulation of lattice polymer models has been investigated since the pioneering works of Stockmayer and Verdier [1] who introduced the concept of micromodifications in the polymer simulation method. This method was later modified and extended to other quasi-crystalline lattices $[2,3]$ and to polymer chains with different topology [4].

Many new ideas have been introduced leading to real acceleration of the simulation processes and enabling one to study long-time dynamic properties of polymer systems [5-9]. However the existing methods do not lead to the final answers which have been asked many times; e.g. whether or not the reptation mechanism dominates in the chain motion or what are the concentration dependencies of polymer dynamics $[2,10]$ ? The answers to these questions can be given by performing large-scale simulations of polymer systems. It is evident that the fast algorithm would accelerate those time consuming simulations.

*To whom correspondence should be addressed; e-mail: prom@chem.uw.edu.pl 
The presented investigations were made using the model polymer chains on a simple cubic lattice. The chains were constructed taking into account the excluded volume effect without long-range attractive interactions (athermal chains). Chains with no excluded volume were also simulated for comparison as well as for checking the validity of the algorithm. The simulations were performed on linear chains consisting of $N=50,100$, and 200 beads.

The classical simulation algorithm used was as follows [2, 8, 11]. During the simulations the chain was modified according to the algorithm by means of the Monte Carlo method and this process was repeated many (order of $10^{6}$ ) times. The set of elementary micromodifications consists of $[8,12]$ :

1. end of chain modifications,

2. 2-bond kink motion,

3. 3-bond kink motion,

4. 3-bond cranckshaft motion.

\section{The simulation algorithm}

Figure 1 shows the idea of micromodification enabling one to change the local conformation of the chain. The process of changing the position of chain bonds was repeated many times - the time scale was defined as proportional to the number of attempted micromodifications per chain length $N$. As was proved, all other motions of the chain are combinations of those elementary modifications [2, 3, 13]. Time unit corresponds to one attempt of every kind of micromodification per one polymer bead.

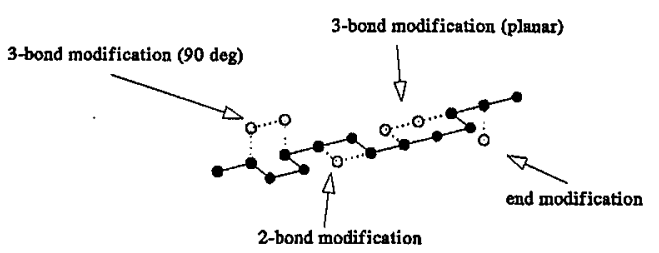

Fig. 1. Scheme of the idea of changing the chain conformation by means of micromodifications in the classical Monte Carlo algorithm for a simple cubic lattice. The full circles represent the old and empty circles represent the new positions of chain beads.

In the proposed algorithm the classical set of elementary motions was replaced by the procedure which enables one to modify the large portion of the polymer chain. The length (number of bonds) $n$ of the chain that is a subject to a substitution was chosen as $n=6$. The choice of $n=6$ was done with respect to the reasonable volume of the set of all possible 6 -bond random walks which could be applied during the modification of the existing chain. The larger value of $n$ (e.g. 8, 10 and so forth) would obviously accelerate the dynamics of the system, however one should expect that the checking of the non-physical motion can be time-consuming as well as the ratio of acceptance of the new configuration can be low. The optimization of such case will be the subject of the forthcoming investigations. Applying the simple idea of the large-scale modification one should realize that there exists the possibility of topological obstacles that could occur during 
the simulation. Such traps would involve the chain into non-physical modifications and therefore lead to false results. The proposed technique enables one to exclude such cases and obtain a correct dynamics of the system under consideration.

In order to understand the problem of the topological traps one should analyze the scheme presented in Fig. 2. The scheme shows a fragment of a chain on a simple cubic lattice (bold line). Now, let us suppose that the fragment of the chain located between beads $k$ and $k+6$ undergoes the elementary motion and the resulting new conformation of this fragment is shown by the dotted line. The basic assumption of such a process is that the connectivity of the chain as well as the geometry of the lattice is maintained during the process. In order to fulfill these requirements there must be no topological obstacles like one shown in Fig. 2 - the vertical fragment of the chain which stays unchanged during the process forbids the formation of the new structure. In order to detect the possibility of occurring of such obstacles we developed the procedure which finds whether or not such a situation can happen. The idea of the detection is simple - one has to check whether or not there are points which can be potentially occupied by a distant (along the chain contour) fragment of the chain and therefore, could block the change of the fragment of the chain into new conformation. Such a point was denoted by $\mathrm{X}$ in the scheme (Fig. 2). The detection of the points, which might cause the intersection, was done by the inspection of the "loop" consisting of the "old" and "new" fragments of the chain. Then the projection of the loop on the three planes $x y, x z$, and $y z$ was done which easily enabled to check whether or not there are any "empty holes", potentially occupied by other chain beads. Should the "holes" be unoccupied - the new conformation was accepted, otherwise it was rejected and the procedure was repeated.

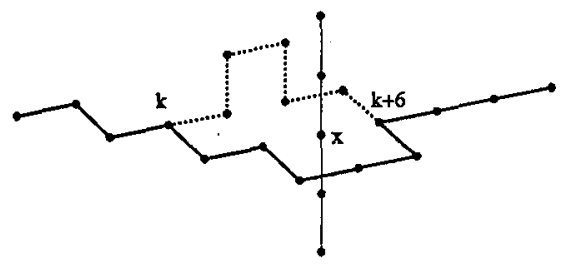

Fig. 2. Scheme of the concept of multi-bond modification of the chain. The $\mathrm{X}$ represents the topological obstacle, which can occur during the modification. The solid line represents the old and dotted line represents the new positions of the chain.

The procedure of the chain modifications was as follows:

a) at first one has to pick at random the bead $k$ of the chain,

b) the fragment of the chain consisting of bonds located between the $k$ and $k+6$ beads is temporarily removed,

c) the new 6-bond fragment of the chain is picked at random from the set of all possible subchains that have the same end-to-end vector $r_{k, k+6}$,

d) then the new 6-bond subchain is built into the modified chain and the excluded volume condition (double occupancy of the lattice points) is checked when necessary; should the test be positive, one has to start the procedure of verifying the topological obstacles during the replacement process, 
e) the lattice points occupied by distant (non-bonded) fragments of the chain causing the topological obstacles are detected and the occupancy test is performed,

f) if the occupancy test (e) is negative, the replacement of the new 6-bond fragment of the chain becomes permanent and the procedure is repeated from (a).

Chain ends also underwent 6 -bond micromodifications but the value of vector $r_{k, k+6}$ does not affect the choice of the new end fragment. Time unit was defined in a similar way as in the classical algorithm: it corresponds to one attempt of chain modification per one polymer bead.

\section{Results and discussion}

The static properties of the chains with and without the excluded volume effect were calculated. The values of mean square end-to-end distance $\left\langle R^{2}\right\rangle$ as well as the mean square radius of gyration $\left\langle S^{2}\right\rangle$ were calculated. The latter parameter was calculated according to the definition

$$
\left\langle S^{2}\right\rangle=\frac{1}{N} \sum_{i=1}^{N}\left\langle\left(r_{i}-r_{\mathrm{cm}}\right)^{2}\right\rangle,
$$

where the averaging is over the Monte Carlo steps and $r_{i}$ is the coordinate vector of the $i$-th bead, $r_{\mathrm{cm}}$ is the coordinate vector of the center of mass.

The values obtained are exactly the same as those obtained in the simulations conducted by the classic algorithm. Parameters $\left\langle R^{2}\right\rangle$ and $\left\langle S^{2}\right\rangle$ scale as $N^{1.18}$ and $N^{1}$ for the model with and without the excluded volume respectively, which is in perfect agreement with previous findings [3, 8]. Also the calculated ratio $\left\langle S^{2}\right\rangle /\left\langle R^{2}\right\rangle$ gave the correct values 0.167 and 0.157 respectively $[4,12]$.

In order to confirm that the proposed algorithm could be useful in the large timescale simulations of the polymer chains it was essential to check whether it produces the correct time-dependent (dynamical) results. In order to test the presented algorithm we performed the calculations of autocorrelation functions, namely a center-of-mass autocorrelation function $g_{\mathrm{cm}}(t)$, an average bead autocorrelation function $g(t)$, and a single-bead autocorrelation function $g_{i}(t)$ defined as follows:

$$
\begin{aligned}
& g_{\mathrm{cm}}(t)=\left\langle\left[r_{\mathrm{cm}}(t)-r_{\mathrm{cm}}(0)\right]^{2}\right\rangle, \\
& g(t)=\frac{1}{N} \sum_{i=1}^{N}\left\langle\left[r_{i}(t)-r_{i}(0)\right]^{2}\right\rangle, \\
& g_{i}(t)=\left\langle\left[r_{i}(t)-r_{i}(0)\right]^{2}\right\rangle,
\end{aligned}
$$

where $r_{i}$ denotes the Cartesian coordinates of the $i$-th polymer bead and $r_{\mathrm{cm}}$ denotes the center-of-mass of the polymer chain.

The example of these functions is given in Fig. 3 and $4 . g_{\mathrm{cm}}(t)$ and $g(t)$ are plotted against time $t$ for a chain consisting of $N=50$ beads without and with the excluded volume effect respectively. The plots were made for the simulations performed according to the classical as well as the new algorithm. One can notice the similar shape of the plots obtained from the two algorithms. Also the slopes of the $g(t)$ and $g_{\mathrm{cm}}(t)$ functions in both cases exhibit the correct values: the average 


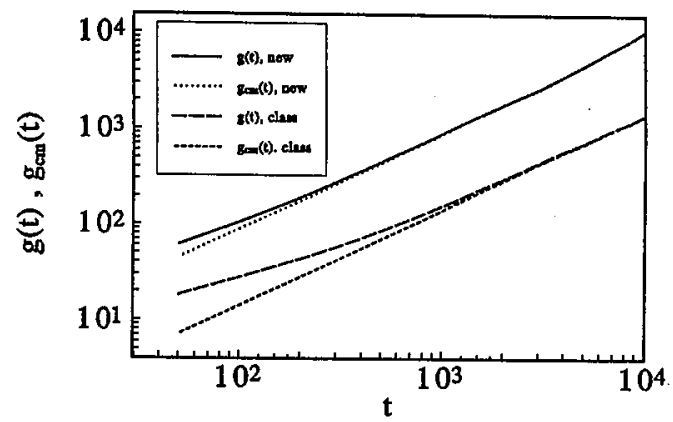

Fig. 3. Plot of autocorrelation functions $g(t)$ and $g_{\mathrm{cm}}(t)$ vs. $t$ for chain consisting of $N=50$ beads without the excluded volume. The results were achieved by the means of the classical (class) as well as the new algorithm (new).

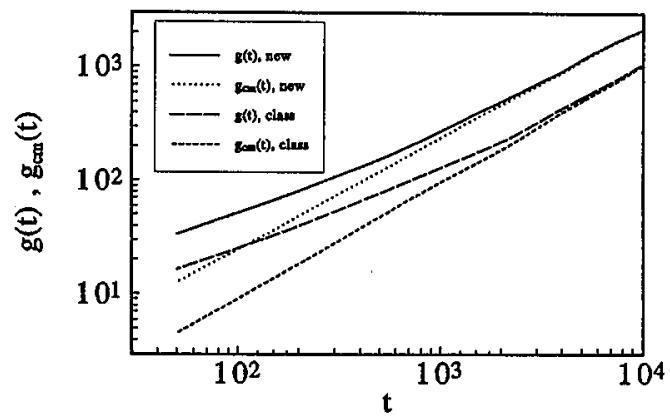

Fig. 4. Plot of autocorrelation functions $g(t)$ and $g_{\mathrm{cm}}(t)$ vs. $t$ for chain consisting of $N=50$ beads with the excluded volume. The results were achieved by the means of the classical (class) as well as the new algorithm (new).

bead autocorrelation function has two different regimes; the center-of-mass autocorrelation function exhibits a constant slope in the whole range of the simulation. The same results were obtained for chains with the excluded volume effect (Fig. 4). The scalings are very close to the Rouse model predictions where $g(t)$ scales as $N^{1 / 2}$ and $N^{1}$, and $g_{\mathrm{cm}}(t)$ scales as $N^{1}$. The scaling obtained from our results shows that our simulation obeys the scaling law and can be used in investigations of the dynamics of polymer chains [2, 12-14].

Another test of consistency of our method was done by calculation of the self-diffusion coefficient $D$ according to the Einstein formula $D=g_{\mathrm{cm}}(t) / 6 t$. According to the well-known method $[2,12]$ we determined values of $D$ from the data taken from the window $2\left\langle S^{2}\right\rangle$ to $10\left\langle S^{2}\right\rangle$, where the dependence of $g_{\mathrm{cm}}(t)$ vs. $t$ is linear. Figure 5 presents the $\log -\log$ plot of the self-diffusion coefficient $D$ vs. number of polymer segments $N-1$ for the chains with the absence of the excluded volume effect. The scaling from our data yielded $(N-1)^{-1.06 \pm 0.07}$ which is in perfect agreement with the theoretical predictions for the Rouse chain $[2,3,10,14]$ where $D \sim(N-1)^{-1}$.

The last test concerned the single-bead autocorrelation function $g_{i}(t)$. In Fig. 6 we present the plot of $g_{i}(t)$ versus the number of the bead $i$ for the case 


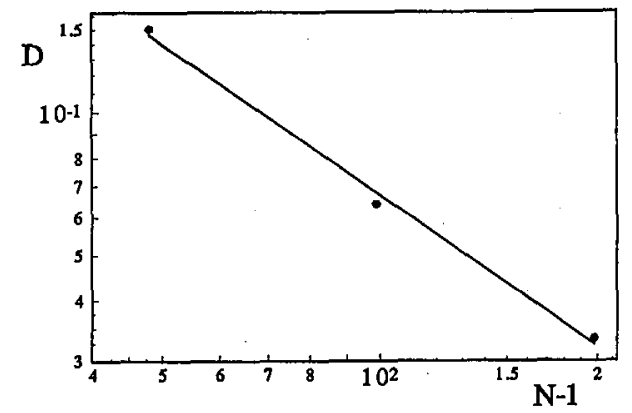

Fig. 5. Plot of the diffusion constant $D$ plotted against the number of beads of the chain $N-1-$ the results are calculated for the case of non-excluded volume simulation using the new algorithm.

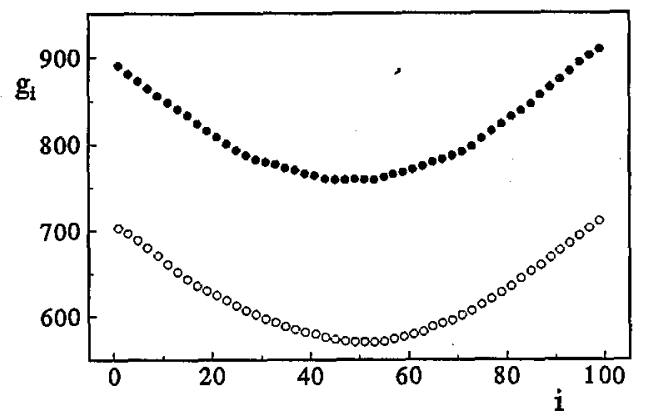

Fig. 6. Example plots of the single bead autocorrelation function $g_{i}(t)$ versus the bead number $i$ - the solid symbols denote the new algorithm, open symbols show results for the classical simulation, non-excluded volume. Data collected for $N=100$, time $t=2500$ (classical) and $t=800$ (new algorithm).

of $N=100$. One can observe that the shapes of the plots are the same for both algorithms studied. Their shapes are also close to that of the Rouse model, which predicts the parabolic dependence $[12,14]$. This result confirms that the frequency of micromodifications and mobility of all beads are proper in the new algorithm.

In order to compare the efficiency of both, the classical and the new fast algorithm we considered the center-of-mass autocorrelation functions obtained by both algorithms. The estimation of the computer time required to obtain a certain displacement of the chain enables one to compare the efficiency of both algorithms. The efficiency of the new algorithm was estimated for two cases: the simulation of polymer chain with and without the excluded volume effect. The latter case does not require the execution of the procedure checking the presence of a topological trap and therefore is much faster. It appeared that the new algorithm is about 3 times faster in the model with the excluded volume and 8 time faster without the excluded volume.

From the results of the above investigations one can conclude that the presented algorithm does really accelerate the simulation process of polymer chains. We expect that the further improvements of this method as well as optimization 
of the length of the fragment of the chain, which is used in the replacement procedure, would make the proposed algorithm more efficient. The application of the new method for more complicated systems, such as polymer melts and concentration dependencies, are in preparation. The algorithm can also be useful for the investigations of the influence of the polymer entanglements on the dynamics of the systems as well as to give some clues which can answer the questions on the mechanism of polymer chain motion.

\section{Acknowledgment}

The support of this work by the University of Warsaw grant BW-1383/25/97 is gratefully acknowledged. The authors express their gratitude for the helpful discussions with Professor Andrzej Koliński.

\section{References}

[1] W.H. Stockmayer, P.H. Verdier, J. Chem. Phys. 36, 227 (1961).

[2] A. Koliński, J. Skolnick, Adv. Chem. Phys. 78, 223 (1990).

[3] K. Kremer, K. Binder, Comput. Phys. Rep. 7, 259 (1988).

[4] A. Sikorski, Macromol. Chem. Theory Simul. 2, 309 (1993).

[5] C.C. Crabb, J. Kovac, Macromolecules 18, 1430 (1985).

[6] J.P. Downey, J. Kovac, Macromolecules 23, 3013 (1990).

[7] J.P. Downey, J. Kovac, Macromolecules 23, 3020 (1990).

[8] A. Sikorski, P. Romiszowski, J. Chem. Phys. 104, 8703 (1996).

[9] P. Romiszowski, W.H. Stockmayer, J. Chem. Phys. 80, 485 (1984).

[10] P.-G. de Gennes, Scaling Concept in Polymer Physics, Cornell University Press, Ithaca (NY) 1979.

[11] A. Koliński, M. Vieth, A. Sikorski, Acta Phys. Pol. A 79, 601 (1991).

[12] A. Koliński, J. Skolnick, R. Yaris, J. Chem. Phys. 86, 309 (1987).

[13] A. Baumgärtner, Ann. Rev. Phys. Chem. 35, 419 (1984).

[14] P.E. Rouse, J. Chem. Phys. 21, 1272 (1953). 